\title{
Salud mental primaria para niños, niñas y adolescentes institucionalizados: perspectiva de equipos de salud
}

\author{
Olga M. Fernández, Ph.D. ${ }^{a}$ \\ Universidad de Chile, Chile \\ Matías Irarrázaval, Mg. ${ }^{b}$ \\ Universidad de Chile, Chile \\ Beatriz Ortega, Psiquiatra. ${ }^{\mathrm{C}}$ \\ Universidad de Valparaíso, Chile \\ Paula Carrasco, Médico. ${ }^{d}$ \\ Universidad de Valparaíso, Chile
}

\author{
Pablo Martínez, Ph.D. ${ }^{\text {e }}$ \\ Universidad de Santiago, Chile \\ Graciela Rojas, Ph.D. ${ }^{f}$ \\ Universidad de Chile, Chile \\ Adrian P. Mundt, Ph.D.9 \\ Universidad de Chile, Chile
}

\section{Olgafernandez@uchile.cl}

\section{Resumen (analítico)}

La salud mental de los niños, niñas y adolescentes institucionalizados en hogares es un desafío clínico y ético para Chile y América Latina. Con el objetivo de explorar experiencias y desafíos percibidos por profesionales de la atención primaria de salud que atienden esta población, se realizó una investigación cualitativa, utilizando un cuestionario con preguntas abiertas. Los datos se analizaron mediante teoría fundamentada. Los resultados mostraron que la atención era parcializada y discontinua. La información sobre el desarrollo vital, la familia, los contextos de vulneración e institucionalización era insuficiente y fragmentada. Además, existía escasa coordinación entre las instituciones que intervienen simultáneamente con los niños, niñas y adolescentes. Se discuten las implicancias para la formación de equipos de salud mental de la atención primaria de salud y la implementación efectiva del trabajo intersectorial y multinivel.

\section{Palabras clave}

Salud mental, salud pública, adolescencia vulnerada.

\section{Thesauro}

Tesauro de Ciencias Sociales de la Unesco.

\section{Para citar este artículo}

Fernández, O. M., Irarrázaval, M., Ortega, B., Carrasco, P., Martínez, P., Rojas, G., \& Mundt, A. P. (2021). Salud mental primaria para niños, niñas y adolescentes institucionalizados: perspectiva de equipos de salud. Revista Latinoamericana de Ciencias Sociales, Niñez y Juventud, 19(2), 1-19. https://dx.doi.org/10.11600/rlcsnj.19.2.4175

\section{Historial}

Recibido: 26.06 .2020

Aceptado: 13.10 .2020

Publicado: 23.04.2021

\section{Información artículo}

El artículo se deriva de un estudio de mayor escala denominado Diseño y pilotaje de un servicio de telepsiquiatría para mejorar la atención de salud mental para niños, niñas y adolescentes con vulneración de derechos y/o sujetos a la ley de responsabilidad penal adolescente. Financiado por Universidad de Chile (Rectoría y Hospital clínico), proyecto n. ${ }^{\circ} 4$ (Concurso de fondos para el desarrollo de telemedicina en el Hospital clínico de la Universidad de Chile). Esta investigación fue realizada entre el 1 de mayo de 2018 y el 30 diciembre de 2018. Área: psicología. Subárea: terapia. 


\section{Primary mental health care for institutionalized children and adolescents: a health team perspective}

Abstract (analytical)

Mental health care for vulnerable and institutionalized children and adolescents is a clinical and ethical challenge in Chile and Latin America. This study explores experiences and challenges encountered when providing mental health care among primary health care professionals. Qualitative research was conducted using an openended questionnaire for interviewing professionals in a rural area of Chile. The data was analyzed using Grounded Theory. The results show a fragmented and discontinuous delivery of care as a large portion of the children's clinical and social history is unknown. There is lack of coordination with other institutions that intervene in this area. The authors discuss implications for primary health care training for children's mental health teams and the need for the implementation of effective integrated and multilevel care systems.

Keywords

Mental health, primary care, disadvantages childhood and adolescence.

\section{Saúde mental primária para crianças e adolescentes institucionalizados: uma visão das} equipes de saúde

Resumo (analítico)

A saúde mental de crianças e adolescentes vulneráveis e institucionalizados é um desafio clínico e ético no Chile e na América Latina. O presente estudo explorou experiências e desafios do cuidado por profissionais da atenção primária à saúde que atendem esta população. A pesquisa qualitativa foi realizada utilizando um questionário de perguntas abertas para entrevistar profissionais da área rural do Chile. Os dados foram analisados usando a Grounded Theory. Os resultados mostraram um atendimento fragmentado e descontínuo. As informações sobre parte da história clínica e social das crianças, e os contextos de vulnerabilidade e institucionalização são insuficientes. Além disso, existia pouca coordenação entre as instituições que intervêm simultaneamente com os crianças e adolescentes. São discutidas implicações para o treinamento de equipes de saúde mental na atenção primária à saúde e para a implementação de sistemas efetivos de atendimento integrado e multinível.

\section{Palavras-chave}

Saúde mental, saúde pública, crianças e adolescentes vulneráveis.

Información autores

[a] Facultad de Medicina Universidad de Chile. Psicóloga, Pontificia Universidad Católica de Chile. Doctora en Psicoterapia, Pontificia Universidad Católica de Chile y Universidad de Chile. Investigadora adjunta Instituto Milenio para la Investigación de la Depresión y la Personalidad (MIDAP). Orcid: 000-0002-3936-6021. H5: 14. Correo electrónico: olgafernandez@uchile.cl [b] Facultad de Medicina Universidad de Chile. Psiquiatra Infantil y de la Adolescencia, Universidad de Chile. Magíster en Salud Pública, Harvard University. Jefe del Departamento de Salud Mental, Diprece/Subsecretaría de Salud Pública, Ministerio de Salud, Santiago, Chile. Investigador adjunto Instituto Milenio para la Investigación de la Depresión y la Personalidad (MIDAP). Orcid: 0000-0002-9087-8305. H5: 10. Correo electrónico: mirarrazavald@gmail.com

[c] Facultad de Medicina Universidad de Chile. Médico, Universidad Católica del Norte, Chile. Psiquiatra Infantil y de la Adolescencia, Universidad de Valparaíso, Chile. Orcid: 0000-0003-2337-0517. H5: 0. Correo electrónico: beaortega@gmail.com [d] Facultad de Medicina, Universidad de Valparaíso, Chile. Médico, Universidad de Valparaíso, Chile. Psiquiatra Infantil y de la Adolescencia, Universidad de Valparaíso, Chile. Orcid: 0000-0002-7170-4318. H5: 1. Correo electrónico: pola.carrasco@gmail.com [e] Departamento de Psiquiatría y Salud Mental, Hospital Clínico Universidad de Chile, Santiago, Chile. Doctor en Psicología, Universidad de Santiago de Chile. Investigador joven en Núcleo Milenio para Mejorar la Salud Mental de Adolescentes y Jóvenes, Santiago, Chile y MIDAP. Orcid: 0000-0003-4482-7993. H5: 8. Correo electrónico: pablo88.martinezdiaz@gmail.com [f] Hospital Clínico J. J. Aguirre, Universidad de Chile. Médico, Universidad Johann W. Goethe, Frankfort del Meno, Alemania. Doctora en Medicina, Universidad Johann W. Goethe, Frankfort del Meno, Alemania. Psiquiatra Adulto, Universidad de Chile. Profesora titular Facultad de Medicina, Universidad de Chile. Orcid: 0000-0002-9577-7415. H5: 18 Correo electrónico: graciela.rojas.castillo@gmail.com

[g] Facultad de Medicina Universidad de Chile y Facultad de Medicina Universidad Diego Portales. Médico Psiquiatra, Doctor Charité Universitätsmedizin, Berlin. Orcid: 0000-0001-8763-4601. H5 19. Correo electrónico: adrian.mundt@uchile.cl 


\section{Introducción}

E n la actualidad se estima que más de 2.7 millones de niños en el mundo viven en dad, abandono por pobreza, abuso en las familias de origen o discapacidad física o mental (Berens \& Nelson, 2015). La evidencia internacional sugiere que los niños, niñas y adolescentes (en adelante NNA) institucionalizados presentan peor salud mental que los NNA que son puestos tempranamente bajo formas de cuidados alternativos más personalizadas y de mejor calidad (Bronsard et al., 2016; Fernández-Daza \& Fernández-Parra, 2013; Humphreys et al., 2015; Sempik et al., 2008), y que la mayoría de ellos reciben atención de salud mental en los servicios de atención primaria (Larsen et al., 2018).

En Latinoamérica y el Caribe alrededor de 200 ooo NNA viven bajo tutela institucional u hogares de protección (Fernández-Daza \& Fernández-Parra, 2013). En Chile, más de 14 mil NNA viven en alguna de las 251 residencias a cargo del Servicio Nacional de Menores (Sename), organismo gubernamental encargado de la protección de derechos de niños y adolescentes vulnerados (Comité de los Derechos del Niño [CRC], 2018). Según datos epidemiológicos chilenos (Vicente et al., 2012), la prevalencia de trastornos mentales en NNA de programas Sename es significativamente más alta que la prevalencia de población general (38.3\%); y según el informe del CRC (2018), el 69.1\% de los NNA en protección por vulneración de derechos presenta al menos un trastorno mental (45.3\% riesgo de suicidio, $40 \%$ dependencia de drogas, $25.7 \%$ trastornos de conducta disocial y $23.5 \%$ negativista desafiante), existiendo una brecha asistencial de $88.9 \%$ para responder a las necesidades de salud mental de esta población. Los recursos humanos especializados en la salud mental de los NNA son insuficientes (Vicente et al., 2012), dificultando el acceso a la atención prioritaria y de calidad para esta población.

Para enfrentar este escenario, en el año 2016, la Subsecretaría de Redes Asistenciales del Ministerio de Salud de Chile (De Ferrari, 2018) estableció un plan de priorización para dar atención de salud integral y oportuna con énfasis en la salud mental para todos 
los NNA de las residencias del Sename, con el fin de volver más efectivo el vínculo entre las residencias y la atención primaria de salud (en adelante APS). Esto implicaba que médicos generales y psicólogos de APS debían resolver los complejos requerimientos de salud mental y enfermedades psiquiátricas en forma prioritaria y oportuna de los NNA vulnerados en sus derechos, trabajando en red con los otros niveles de atención en salud e intersectorialmente. Dada la gravedad de las enfermedades de salud mental que presenta este grupo específico de NNA y la sobrecarga a estos equipos de salud (Arón \& Llanos, 2004; Scharager \& Molina, 2007; Vilà et al, 2015) es una necesidad y requerimiento activar las consultorías en salud mental (Kirsten et al., 2017; Minoletti et al., 2012); ello con el objetivo de optimizar la capacidad resolutiva de estos equipos de atención primaria y de mejorar la oportunidad, pertinencia y calidad del diagnóstico, tratamiento, rehabilitación y continuidad de cuidados de las personas con problemas de salud mental atendidas en la red de salud. Lo anterior, a través de un trabajo articulado y planificado entre los equipos del nivel primario de atención y los equipos especializados de salud mental (Minoletti et al., 2012). En este mismo sentido, el desarrollo de la telemedicina - específicamente la telepsiquiatría - se constituye en una herramienta útil para facilitar la realización y continuidad de las consultorías en salud mental, sobre todo al garantizar el acceso a distancia a servicios de salud mental especializados (Hilty et al., 2013). Al incorporar la atención en salud mental a los NNA en sistemas de protección en la APS, se hace necesario conocer las características específicas y diferenciales de este tipo de atenciones no realizadas prioritariamente por estos equipos de salud de APS. Al respecto, es necesario mencionar que la población de NNA en sistemas de protección es excluida normalmente de muchos estudios por la dificultad de obtener los consentimientos de participación por parte de sus padres o apoderados, por lo cual los datos son extremadamente escasos (MacMillan et al., 2007). Es relevante, entonces, acercarse a las experiencias de atención desde los tratantes de modo de poder contar con información que nutra a consultorías, teleconsultorías y actividades de formación, con pertinencia y efectividad, así como que sean atingentes a las necesidades percibidas por los propios ejecutores de las intervenciones.

En este contexto general, y en el marco de un proyecto de investigación cuyo propósito es diseñar un servicio de consultoría que responda a las necesidades de los equipos de salud mental de APS que atienden a NNA institucionalizados, la pregunta que surge es: ¿cuáles son las experiencias y necesidades percibidas subjetivamente por los equipos de salud en la práctica directa de atención estos NNA? Se utiliza un diseño cualitativo porque se busca recoger lo más vívidamente posible la experiencia subjetiva y las características particulares de los contextos APS, ya que no se dispone de información directa 
al respecto. Así, el estudio tiene como objetivo explorar las experiencias y necesidades percibidas subjetivamente por los profesionales de los equipos de salud mental de APS en la atención de salud mental para NNA institucionalizados con el propósito de motivar el desarrollo de conocimiento específico en relación a las particularidades de la atención en salud mental de NNA en esta condición, que aporte a la calidad y eficiencia a los servicios de consultoría y teleconsultoría, mejorando la calidad y eficacia de la atención de los NNA vulnerados en sus derechos.

\section{Método}

El diseño de esta investigación fue transversal, de tipo descriptivo-relacional y de carácter cualitativo. La metodología cualitativa permite abordar los problemas de salud que requieren incorporar la subjetividad de los sujetos investigados para lograr un análisis e implementación adecuada al contexto social y subjetivo de los participantes (Amezcua \& Gálvez, 2002).

\section{Participantes}

Los participantes fueron el $100 \%(n=8)$ de los profesionales de los equipos de salud mental de dos centros de atención primaria de una zona rural de Chile (V región de Chile), que aceptaron ser parte de un estudio más amplio (Diseño y pilotaje del servicio de telepsiquiatría, y que atienden a NNA con vulneración de derechos). Estos centros de APS se eligieron por criterios de accesibilidad y conveniencia determinados por las condiciones necesarias para la implementación del estudio de telepsiquiatría en el cual se inserta este estudio. Los centros cumplían los siguientes criterios: 1 ) presencia de equipo de salud mental; 2) implementación en curso del plan de priorización en atención de salud integral y oportuna, con énfasis en la salud mental, para todos los NNA de las residencias del Sename; 3) experiencia en consultorías de salud mental; 4) trabajo colaborativo anterior con profesionales de los equipos de salud mental; y 5 ) aprobación del Comité de Ética del Servicio Regional de Salud y de los directores de cada uno de los consultorios para participar de esta investigación.

Los profesionales participantes, a su vez, debían cumplir los siguientes criterios: 1) atender o haber atendido NNA con vulneración institucionalizados en este consultorio por problemas de salud mental en los últimos seis meses; 2) firmar consentimiento 
informado de participación. Fueron invitados a participar ocho profesionales que cumplían estos criterios, de los cuales el 100\% aceptó (tabla 1).

\section{Tabla 1}

Descripción de los participantes

\begin{tabular}{ccccc}
\hline Entrevista & Profesión & $\begin{array}{c}\text { Experiencia profesional } \\
\text { APS (años o meses) }\end{array}$ & Género & Establecimiento \\
\hline 1 & Médico/a & 5 años & $\mathrm{F}$ & CECOSF* $^{*}$ \\
2 & Asistente social & 6 años & $\mathrm{F}$ & CECOSF* $^{*}$ \\
3 & Médico/a & 1 año & $\mathrm{F}$ & Consultorio \\
4 & Psicólogo/a & 16 años & $\mathrm{F}$ & Consultorio \\
5 & Médico/a & 2 años & $\mathrm{F}$ & Consultorio \\
6 & Psicólogo/a & 6 años & $\mathrm{F}$ & CECOSF* \\
7 & Psicólogo/a & 8 meses & $\mathrm{F}$ & Consultorio \\
8 & Psicólogo/a & 3 años & $\mathrm{F}$ & Consultorio \\
\hline
\end{tabular}

Nota. *Centro Comunitario de Salud Familiar.

\section{Procedimientos}

Se contactaron potenciales participantes mediante correo electrónico y teléfono, se les explicó el propósito de la entrevista y se les entregó información del proyecto y el consentimiento informado. Se utilizó un cuestionario de preguntas abiertas, contestado vía online (tabla 2). El cuestionario comienza con una pregunta inicial muy amplia y luego se pregunta directamente acerca de: 1) problemas de salud mental más frecuentes que traen a los NNA a consultar; 2) situaciones complejas durante la entrevista y temas difíciles de explorar; $\mathrm{y}, 3)$ casos difíciles de diagnosticar, uso de fármacos y manejo de contingencias ambientales.

\section{Tabla 2}

\section{Cuestionario para profesionales}

1. Comente libremente acerca de su experiencia de atención en el consultorio de niños, niñas y adolescentes que vienen de los centros del Sename por problemas de salud mental.

2. Generalmente, ¿qué o cuáles son los problemas por los que vienen a consultar?

3. ¿Cuáles son los aspectos y situaciones más difíciles de manejar durante la atención?

4. ¿Qué tipos de problemas son los más complejos de diagnosticar y tratar según su experiencia?

5. ¿Le gustaría comentar algo más? 


\section{Análisis de datos}

Siguiendo los procedimientos de la teoría fundamentada (Soneira, 2006; Strauss \& Corbin, 2002), dos investigadores, en forma paralela e independiente, codificaron las respuestas del cuestionario con el sistema de codificación abierta, para luego conciliar las categorías iniciales emanadas. Cuando no hubo consenso en la clasificación, se procedió a la triangulación con un tercer investigador para lograr un acuerdo intersubjetivo. A partir de la codificación abierta, se construyeron las características descriptivas acerca de las atenciones en salud mental a los NNA institucionalizados. Luego, se articularon las categorías y conceptos de forma relacional interpretativa, a modo de generar un fenómeno comprensivo axial (Soneira, 2006; Strauss \& Corbin, 2002). Para asegurar la rigurosidad de los resultados, las interpretaciones fueron realizadas a través de un acuerdo intersubjetivo del equipo investigador completo.

\section{Resultados}

El análisis descriptivo permitió extraer tres categorías: 1) características de la atención; 2) motivos de consulta o problemas más frecuentes; 3) dificultades percibidas en la atención. Finalmente se presenta un fenómeno central relacional que integra las categorías.

\section{Características de la atención}

Según los profesionales, los cuidadores que traen a los NNA a consultar desconocen en gran parte la historia personal y familiar de los consultantes, por lo que las atenciones se realizan con anamnesis incompletas. Además, se desconoce la historia institucional del niño: las razones de su institucionalización, los tiempos de permanencia en esta residencia o en otras anteriores. Esta falta de contexto dificulta la comprensión psicosocial y evolutiva del caso, afectando la calidad de las intervenciones. Los niños - como pacientes- se caracterizan por ser desconfiados en el contacto y en la información que entregan; presentan dificultad de vincularse con el tratante y son poco receptivos a sus preguntas y comentarios, resultando difícil acceder a lo que realmente les sucede o les preocupa. Por su parte, los tratantes confiesan que la atención de estos NNA tiene un alto impacto emocional para ellos; por una parte genera dolor, frustración y cansancio y, por otra parte, gran satisfacción por los logros alcanzados en algunas circunstancias. 
Los niños que he atendido son de [nombre institución]; asisten en compañía del apoderado encargado de cada casa, presentando como dificultad inicial el desconocimiento de antecedentes familiares patológicos mentales, antecedentes patológicos del paciente, además del porqué se encuentran en la institución, generando en primera instancia una anamnesis incompleta, interfiriendo así en el adecuado manejo. (Entrevista 1, E1)

\section{Motivos de consulta o problemas más frecuentes}

Los motivos de consulta o problemas por los que se solicita atención son dificultades en la interacción social en la institución o en el establecimiento, vinculadas al manejo de emociones (rabia, irritabilidad) y respeto de límites y normas, llegando a conductas violentas hacia pares, adultos u objetos. Además, se mencionan los problemas asociados a maltrato o abuso sexual, histórico o reciente. En términos sintomáticos o de diagnósticos se mencionan: trastorno oposicionista desafiante, síndrome por déficit atencional, conductas autolesivas (autolesiones, ideación e intentos de suicidios) y, en una menor proporción, reacciones ansiosas y ánimo depresivo. Los episodios psicóticos, trastorno afectivo bipolar y del espectro autista, se presentan esporádicamente. Por otra parte, se observa la pseudoconsulta a la APS, que tiene como objetivo buscar una derivación a un centro de mayor complejidad dentro del sistema de atención; como lo dice este profesional: «Sí, me han llegado, pero más que nada para lograr la interconsulta a la atención secundaria, la mayoría de ellos no mantiene el control en atención primaria» (E5). Esto sucede especialmente cuando los NNA han recibido diagnóstico y medicación desde otros sistemas de atención y buscan dar continuidad de la atención ingresando al sistema público.

Por otra parte, llama la atención que los tratantes no se refieren a necesidades o motivos de consulta planteados por los NNA. La conceptualización del problema o del motivo de consulta se refiere a un problema registrado y definido por otro, externo al NNA (instituciones de protección o escuela). En otras ocasiones, el motivo de consulta se homologa inmediatamente a una categoría diagnóstica que explique los comportamientos descritos por los adultos. No hay mención a las necesidades, malestares y dificultades planteadas por los NNA como motivo de consulta desde su perspectiva subjetiva o experiencia de malestar.

\section{Dificultades percibidas en la atención}

Las dificultades percibidas en la atención a los NNA se presentan organizadas en tres áreas: del profesional tratante, del paciente y dificultades en el contexto de atención y de institucionalización. 
Los profesionales hacen referencia a su falta de conocimientos en salud mental para diagnósticos más precisos, diferenciales y morbilidades asociadas: «En este sentido, la formación de pregrado es insuficiente» (E3). «También es difícil manejar el tema de las comorbilidades...; qué me pasa a mí cuando veo niños con déficit atencional, cuando tienen comorbilidades; ahí también se hace mucho más complejo» (E5). Además, mencionan que les faltan habilidades para mantener una entrevista logrando un mejor vínculo y motivación con el paciente, así como también con los adultos responsables: «No puedo acceder a él» (E6). «¿Cuáles serán sus vivencias?» (E3). [Qué es difícil para ti] «muchas veces el tema de abordar a los papás o al cuidador» $\left(\mathrm{E}_{5}\right)$.

En relación con las dificultades en la atención aportadas por los pacientes, los equipos hacen referencia a la actitud hacia la consulta: poco comunicativos, no muestran interés en lo que se les pregunta, no hablan casi nada. Además, mencionan dificultades de adherencia a los controles y resistencia a la ingesta de medicamentos: «No quieren contar de nuevo su historia, se muestran aburridos en la atención, poca motivados a cooperar» (E3). «No quieren tomar los medicamentos» (E1). Unido a lo anterior, se percibe también una dificultad para establecer el vínculo cercano y de confianza con los profesionales: «Se hace complejo realizar un trabajo vincular con los NNA; muchas veces están sobreintervenidos por diversos dispositivos» (E6).

En las dificultades percibidas en el contexto de atención se observan aquellas aportadas por el centro/sistema de salud y las relacionadas con el contexto institucional de los niños. Los centros de salud presentan barreras administrativas institucionales, tales como escasas horas disponibles de atención para salud mental, baja frecuencia entre consultas y tiempos de atención muy breves, lo que genera una respuesta de atención retardada o no atingente al problema. Además, los centros de salud presentan barreras logísticas (cambios de lugares y horarios de atención, alta rotación o movilidad de recursos humanos), lo que afecta la continuidad y la calidad de las intervenciones:

Para este tipo de casos el rendimiento de treinta minutos es igual que para todos los otros casos [de morbilidad aguda general]; sin embargo, la evaluación de ingreso [de morbilidad de salud mental] suele requerir más tiempo, lo que se traduce en mayor dificultad para trabajar adecuadamente. (E3)

La institución responsable del NNA también puede llegar a interferir en la atención exitosa en salud mental. El encargado de traer al NNA a consultar no siempre es quien más conoce al paciente: desconoce su historia individual y familiar pasada y presente; no 
puede aportar observaciones más detalladas de los problemas actuales y tampoco será quién deba implementar las indicaciones sugeridas por el especialista. Por las características del funcionamiento de las instituciones de protección resulta difícil sensibilizar acerca de la complejidad del problema y de las indicaciones específicas de manejo. A su vez, las instituciones que albergan a los NNA no promueven la coordinación y complementariedad entre las distintas intervenciones que reciben los NNA desde los distintos sistemas de apoyo (judiciales, escolar, familiares y sociales), de modo que la atención de salud queda como una acción no articulada. También se menciona que la participación e involucración de los padres en los tratamientos es muy necesaria, pero se hace muy difícil por no vivir con sus hijos y por las situaciones judiciales asociadas.

Asisten en compañía del apoderado encargado de cada casa, presentando como dificultad inicial el desconocimiento de antecedentes familiares mentales, antecedentes patológicos del paciente, además del porqué se encuentran en la institución. (E1)

Cuando se relacionan con abuso sexual infantil, asisten por sintomatología asociada al evento, a pesar de que la reparación se encuentre interviniendo en otros centros especializados. $\left(\mathrm{E}_{4}\right)$

\section{Fenómeno central}

Del análisis relacional de la información descriptiva se construye un fenómeno interpretativo axial central que se ha llamado «atención sin memoria y con deseo (frustrado)» (figura 1), que permite crear un modelo comprensivo de la situación.

Este fenómeno da cuenta de la atención a un NNA sin historia en el momento de ser atendido. El cuidador que lo lleva a consultar no aporta antecedentes personales (evolutivos, familiares, intervenciones, entre otras) pasados y presentes; generalmente se centra en el problema puntual que motivó la consulta. El paciente tampoco aporta información acerca de sí; colabora poco en las entrevistas; asiste, pero no muestra interés en el servicio que se le quiere brindar; se desconoce qué siente o qué piensa sobre estar ahí. El NNA asiste sin motivación propia y muchas veces desinformado de la situación y del objetivo de la consulta; generalmente ha asistido en otras ocasiones a consultas similares sin resultados y no está dispuesto a colaborar con un desconocido, ni menos compartir sus experiencias personales. A su vez, el escaso tiempo de la consulta tampoco permite profundizar y explorar con detención en el caso, y la siguiente cita será muy distanciada, lo que genera que en el nuevo encuentro el NNA se habrá transformado en un desconocido nuevamente: no hay memoria del NNA. 
Figura 1

Fenómeno relacional «sin memoria y con deseo (frustrado)»

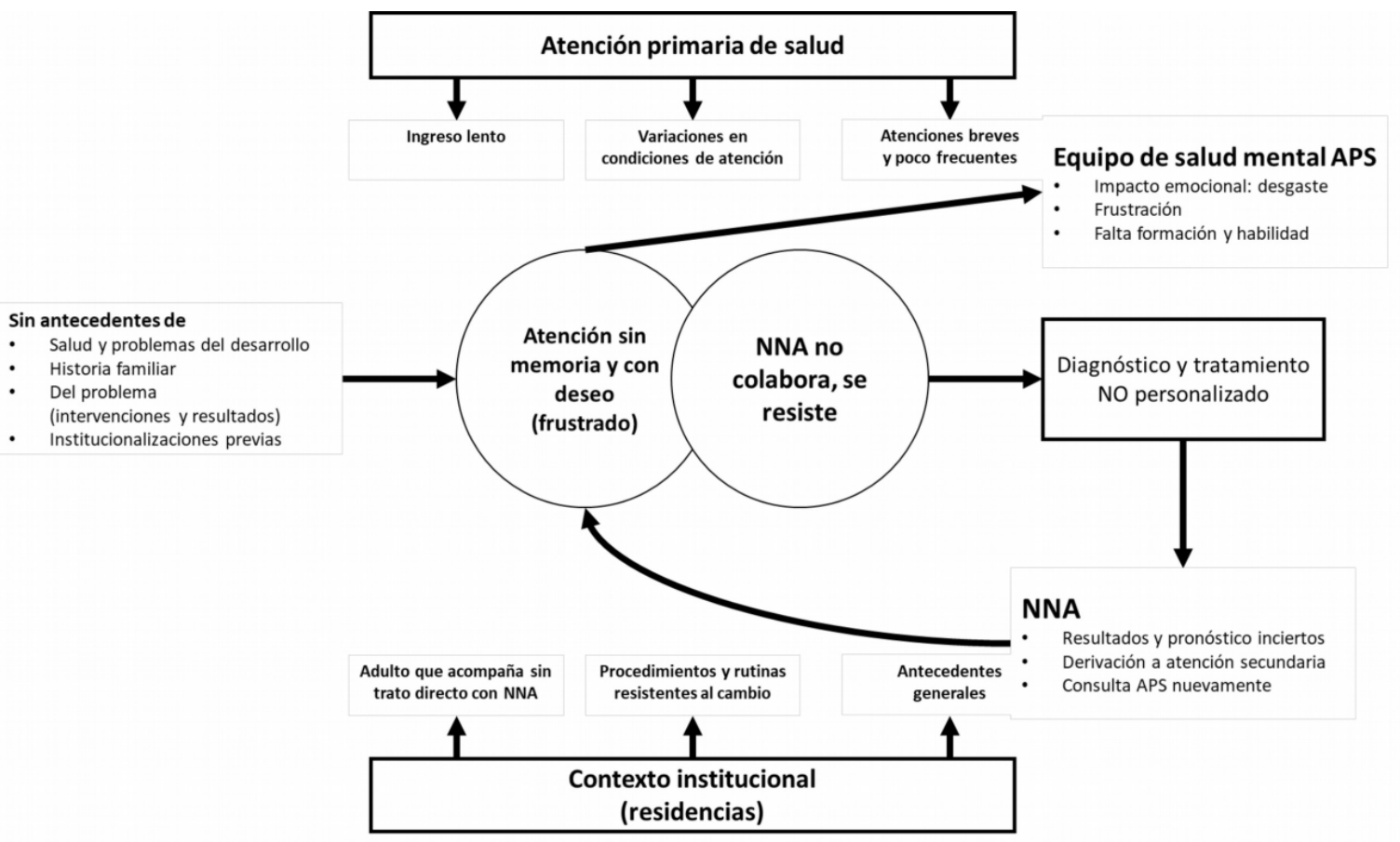

El tratante quiere ayudar, se siente interpelado/desafiado a intervenir, pero siente falta de información, de conocimientos y habilidades. Los equipos perciben que no logran comprender realmente al NNA; no pueden distinguir con claridad los signos necesarios (semiología insuficiente) y se desconoce lo que realmente piensa o siente el NNA de primera fuente.

En esta situación de incertezas, se deben realizar las intervenciones (farmacológicas o psicosociales) o solicitar derivación. Estos profesionales se encuentran atrapados entre las exigencias de atención de la institución, por una parte, y su deseo (incumplido) de estar al servicio del niño. El NNA probablemente es diagnosticado y tratado sin la especificidad necesaria a sus necesidades y contexto, aumentando las probabilidades de resultados inciertos. El profesional no puede centrarse realmente en el niño presente; su trabajo termina siendo más un marco formal que un apoyo efectivo a la salud mental del paciente.

Finalmente, no hay certeza de la continuidad de la atención. Los NNA pueden haber sido trasladados a otra institución de mayor complejidad, o las instituciones optan por atención en salud mental fuera del sistema público, situación que no es sostenible en el tiempo por los recursos que eso implica. El profesional del consultorio también aporta 
discontinuidad, dado que la rotación en los equipos de salud mental es alta por razones laborales, económicas y de desgaste emocional.

\section{Discusión}

Los resultados anteriormente presentados responden a la interrogante acerca de las experiencias y necesidades percibidas subjetivamente por los equipos de salud en la práctica directa de atención estos NNA institucionalizados. La atención es percibida como parcializada y discontinua, con falta de antecedentes del NNA, su familia y su historia, lo que limita la comprensión del caso y el diseño de un tratamiento adecuado. Los equipos de APS reconocen la complejidad psicopatológica y, especialmente, los aspectos psicosociales involucrados en la atención de los pacientes. Los tratantes aluden a la complejidad y desgaste en este tipo de atenciones, a la necesidad de especialización para trabajar con este grupo específico y a las problemáticas éticas asociadas a un tratamiento escasamente eficiente.

Los resultados del estudio son coincidentes con lo planteados por otros autores (Esponda et al., 2019; Ståhlberg et al., 2010) que destacan que es esencial para los profesionales de salud mental de APS contar con un apoyo real y efectivo de especialistas en el tema, así como capacitación que les permita mejorar sus competencias efectivas; ello debido a este tipo de atenciones requiere de especialización ya que, de lo contrario, surge el dilema ético de estar proporcionando a saber un tratamiento deficiente.

La necesidad de apoyo técnico y emocional a los equipos que trabajan con NNA institucionalizados ha sido reportada por otros estudios chilenos (Arón \& Llanos, 2004; Santana \& Farkas, 2007) e internacionales (Huertas, 2005; Olabarría \& Mansilla, 2007; Sánchez-Reyes et al., 2019), aludiendo al desgaste profesional, que a la vez se relaciona con la alta rotación en los equipos de salud de este tipo de población. El cambio de tratantes reedita situaciones de abandono y victimización de su historia vital, aumentando sentimientos de desconfianza y desesperanza ante la ayuda profesional (Hueche et al., 2019).

Para el diseño de un sistema de atención de salud mental para NNA institucionalizados se hace necesario mejorar diversos aspectos de la atención. Es necesario diseñar mecanismos que permitan mejorar la falta de información clínica y psicosocial al momento del ingreso, para lo cual la solicitud de informes o comunicaciones breves con los tratantes o director(a) de la residencia puede ayudar a reducir esta brecha y aumentar la 
capacidad resolutiva del equipo. La coordinación entre equipos también es considerada un elemento esencial, de tal forma que los profesionales de APS puedan trabajar coordinadamente con los cuidadores y organizaciones que están entregando servicios de apoyo, y puedan obtener una evaluación comprensiva del contexto ecológico además de la compleja historia de salud médica y mental (Berens \& Nelson, 2015). Esto es fundamental para asegurar que no se cumpla la ley inversa de cuidados según la cual los grupos más vulnerables y con sintomatología más compleja son quienes tienen un menor acceso a los servicios de salud mental (Lorenc et al., 2013; Santos et al., 2019). Una atención comprensiva e integrada con los otros sectores involucrados tiene el potencial de actuar como red de protección y resguardo de los NNA vulnerados en sus derechos, ante las inequidades en la atención de salud, mejorando así la entrega de servicios según las necesidades médicas y psicosociales (Ministerio de Salud, 2018). Por la complejidad de los pacientes en esta población se podría considerar la atención en duplas (médico-psicólogo o asistente social) y de ampliar la duración estándar de entrevista de salud mental en la APS, a modo de dar una atención más integral y coordinada, en relación a las necesidades y motivaciones del NNA.

Los profesionales de APS entrevistados describen el escaso entrenamiento para manejar entrevistas de salud mental, lo que plantea la necesidad de integrar con mayor énfasis la formación en competencias para el trabajo infanto-adolescente en los programas de formación de los profesionales de la salud. A la vez, es necesario desarrollar las capacidades para diferenciar a los NNA que pueden verse beneficiados por la atención en APS y los que necesitan servicios más especializados (Larsen et al., 2018). En este contexto, Rozanski (2003) plantea que se debieran evitar posibles daños secundarios (revictimización) con intervenciones por parte de profesionales no capacitados para atender un perfil de usuario como este. Aquí cobran relevancia las implicancias éticas que tiene la atención de NNA bajo el sistema de protección, considerando que han vivido experiencias adversas a temprana edad y presentan una alta prevalencia de problemas de salud mental. Existe evidencia que respalda los efectos del estrés tóxico a temprana edad y su impacto en el neurodesarrollo, generando cambios a nivel de las estructuras cerebrales que regulan la respuesta al estrés y sus mecanismos de respuesta neuroendocrinos (Hambrick et al., 2019; Jacoby et al., 2016). Publicaciones sobre las consecuencias de la crianza en sistemas institucionales también dan cuenta de las consecuencias del - ya mencionado- estrés tóxico a corto y largo plazo, además de un importante deterioro psicológico y conductual (Forkey \& Szilagyi, 2014; Nelson III et al., 2019). Para revertir esta situación se han elaborado guías para la evaluación y cuidados en salud para niños institucionalizados, ya que 
se plantea que la interacción de un proveedor de la salud con formación en trauma puede ser transformadora para los usuarios (Forkey \& Szilagyi, 2014).

Por su parte, los problemas asociados a la fragmentación de servicios apoyan la importancia de crear una red de proveedores y servicios con experiencia en el tratamiento de NNA de alto riesgo psicosocial. Existe evidencia que la construcción de alianzas entre los diferentes sectores utilizando un modelo integrativo permite el cuidado efectivo y eficiente que requieren los NNA con vulneración de derechos (Zachik et al., 2010). El modelo de atención comunitaria del Plan Nacional de Salud Mental apoya esta integración y reconoce el valor de la mejoría en la navegación de los pacientes entre los componentes del servicio de salud y la coordinación de los diferentes proveedores (Ministerio de Salud, 2017). Los indicadores basados únicamente en el número de casos atendidos o derivaciones realizadas son parciales e insuficientes para considerar dar respuesta a las necesidades y derechos de los NNA en salud (Durán-Strauch et al., 2011). El modelo debiera considerar disminución del malestar sintomático y subjetivo, calidad de vida y satisfacción, utilizando evaluaciones cuantitativas y cualitativas que consideren las percepciones de los actores del proceso sanitario: NNA y profesionales tratantes.

Las situaciones anteriormente descritas muestran que el sistema de salud otorga una atención focalizada en las necesidades del sistema y no necesariamente en las expectativas y necesidades de los NNA, lo cual interroga respecto al sentido mismo de dicha atención. En este contexto, es necesario implementar el modelo de atención integral en salud para responder a las expectativas de atención, mediante estrategias que permitan humanizarla y el acompañamiento de los NNA. La humanización de la atención en salud hace referencia a una confrontación entre una visión que privilegia el valor de la eficacia basada en los resultados de la tecnología y la gerencia y, por otro lado, una visión que otorga primacía al respeto por el ser humano, su autonomía y la defensa.

El presente estudio también tiene algunas limitaciones. El tamaño muestral en la investigación cualitativa generalmente se encuentra dado por el criterio de saturación y no por una cantidad de sujetos en particular (Martínez-Salgado, 2012). Sin embargo, haber contado con un mayor número de participantes en el área de la salud en centros APS hubiera otorgado mayor potencia a los hallazgos. Los datos obtenidos probablemente representan desafíos que se encuentran a lo largo del país y posiblemente en otros países latinoamericanos. No obstante, debido a su foco exclusivo en un servicio de salud regional particular y la heterogeneidad evidente en la distribución de recursos de salud mental en el país (Ministerio de Salud, 2017), la generalización a nivel país podría ser limitada. 
Más aún, las diferencias estructurales en los sistemas de salud mental limitan de manera considerable la generalización al resto de América Latina. Sin embargo, la información obtenida instituye un aporte al conocimiento específico de la atención clínica de NNA con alta vulnerabilidad psicosocial, considerando la escasa información disponible al respecto. Un desafío para futuras investigaciones es explorar las percepciones de atención en salud mental en APS de los NNA y de las instituciones solicitantes del servicio, a modo de enriquecer la comprensión del fenómeno, integrando las visiones de todos los implicados. También sería interesante evaluar el impacto de consultorías especializadas y de capacitaciones específicas en el tema, en la calidad y resultados de la atención desde la perspectiva de todos los actores.

Finalmente, hay que mencionar que este es el primer estudio de esta naturaleza en Chile, el cual recoge las percepciones de profesionales de centros de salud familiar respecto a la atención de esta población específica. Esta información espera dar mayor relevancia y atingencia a las capacitaciones y consultorías, así como también diseñar modelos de atención en salud mental más específicos para NNA dadas las necesidades y desafíos que experimentan los equipos de salud mental de la APS. Se requiere un entrenamiento adecuado de los trabajadores de salud mental, recursos y tiempo adicionales para la coordinación de los casos, mejorar los sistemas de comunicación entre servicios para el acceso oportuno a la información que requieren los diversos actores involucrados en la atención del NNA.

En conclusión, la atención de salud mental primaria dirigida a NNA en sistemas de protección no puede ser realizada sin memoria y con deseo (frustrado); es por esto que se deben integrar importantes apoyos para la vinculación efectiva de los establecimientos de APS y las residencias, las mejoras en los sistemas de información y en las ofertas de capacitación en salud mental deben ser un complemento necesario a la consultoría de salud mental regular.

\section{Agradecimientos}

Este estudio fue financiado por el Fondo de Subvenciones para el desarrollo de proyectos de telemedicina en el Hospital Clínico de la Universidad de Chile. Además, fue apoyado por ANID: Iniciativa Científica Milenio/Instituto Milenio para la Investigación en Depresión y Personalidad-MIDAP ICS13_005/Núcleo de Milenio Mejorar la 
salud mental de los adolescentes y jóvenes-IMHAY NCS17_035; y FONDECYT Regular 1190613 .

\section{Referencias}

Amezcua, M., \& Gálvez, A. (2002). Los modos de análisis en investigación cualitativa en salud: perspectiva crítica y reflexiones en voz alta. Revista Española de Salud Pública, 76(5), 423-436. https://doi.org/10.1590/s1135-57272002000500005

Arón, A. M., \& Llanos, M. T. (2004). Cuidar a los que cuidan: desgaste profesional y cuidado de los equipos que trabajan con violencia. Sistemas Familiares, 2o(1-2), 5-15.

Berens, A. E., \& Nelson, C. A. (2015). The science of early adversity: Is there a role for large institutions in the care of vulnerable children? The Lancet, 386(9991), 388-398. https://doi.org/10.1016/So140-6736(14)61131-4

Bronsard, G., Alessandrini, M., Fond, G., Loundou, A., Auquier, P., Tordjman, S., \& Boyer, L. (2016). The prevalence of mental disorders among children and adolescents in the child welfare system: A systematic review and meta-analysis. Medicine, 95(7). https://doi.org/10.1097/md.0000000000002622

Comité de los Derechos del Niño [CRC]. (2018). Informe de la investigación relacionada en Chile en virtud del artículo 13 del Protocolo facultativo de la Convención sobre los Derechos del Niño relativo a un procedimiento de comunicaciones. https://bit.ly/39zagKT

De Ferrari, M. I. (2018). Informe de gestión Sename de la Subsecretaría de Redes Asistenciales: años 2016-2018. Subsecretaría de Redes Asistenciales, Ministerio de Salud, Gobierno de Chile.

Durán-Strauch, E., Guáqueta-Rodríguez, C. A., \& Torres-Quintero, A. (2011). Restablecimiento de derechos de niños, niñas y adolescentes en el sistema nacional de bienestar familiar. Revista Latinoamericana de Ciencias Sociales, Niñez y Juventud, 9(2), 549-559.

Esponda, G. M., Hartman, S., Qureshi, O., Sadler, E., Cohen, A., \& Kakuma, R. (2019). Barriers and facilitators of mental health programmes in primary care in lowincome and middle-income countries. The Lancet Psychiatry, 7(1), 78-92. https:// doi.org/10.1016/S2215-0366(19)30125-7

Fernández-Daza, M. P., \& Fernández-Parra, A. (2013). Problemas de comportamiento y competencias psicosociales en niños y adolescentes institucionalizados. Universitas Psychologica, 12(3), 797-810. https://doi.org/10.11144/javeriana.upsy12-3.pccp 
Forkey, H., \& Szilagyi, M. (2014). Foster care and healing from complex childhood trauma. Pediatric Clinics, 61(5), 1059-1072. https://doi.org/10.1016/j.pcl.2014.06.015

Hambrick, E. P., Brawner, T. W., Perry, B. D., Brandt, K., Hofmeister, C., \& Collins, J. O. (2019). Beyond the ACE score: Examining relationships between timing of developmental adversity, relational health, and developmental outcomes in children. Archives of Psychiatric Nursing, 33(3), 238-247. https://doi.org/f6vg

Hilty, D. M., Ferrer, D. C., Parish, M. B., Johnston, B., Callahan, E. J., \& Yellowlees, P. M. (2013). The effectiveness of telemental health: A 2013 Review. Telemedicine Journal and e-Health, 19(69), 444-454. https://doi.org/10.1089/tmj.2013.0075

Hueche, C., Lagos, G., Ríos, N., Silva, E., \& Alarcón-Espinoza, M. (2019). Vínculos afectivos en adolescentes institucionalizados, Chile. Revista Latinoamericana de Ciencias Sociales, Niñez y Juventud, 17(2), 1-19. https://doi.org/10.1160o/1692715x.17217

Huertas, M. (2005). Aproximación a la dinámica riesgos-autocuidado en equipos de operadores sociales que trabajan con desplazados en pasto, Colombia. Psykhe, $14(2)$, 133-147. https://doi.org/10.4067/so718-22282005000200011

Humphreys, K. L., Gleason, M. M., Drury, S. S., Miron, D., Nelson, C. A., Fox, N. A., \& Zeanah, C. H. (2015). Effects of institutional rearing and foster care on psychopathology at age 12 years in Romania: Follow-up of an open, randomised controlled trial. The Lancet Psychiatry, 2(7), 625-634. https://doi.org/10.1016/S2215-0366(15)00095-4 Jacoby, N., Overfeld, J., Binder, E. B., \& Heim, C. M. (2016). Stress neurobiology and developmental psychopathology. Developmental Psychopathology, 2, 1-45. https://doi.org/gd8zzk

Kirsten, K., Acuña, J., Huepe, G., \& Fuenzalida, M. (2017). Programa de desarrollo de salud mental para la atención primaria: Prodesam. Revista Gaceta de Psiquiatría Universitaria, 13(2), 179-192.

Larsen, M., Baste, V., Bjørknes, R., Myrvold, T., \& Lehmann, S. (2018). Services according to mental health needs for youth in foster care?-A multi-informant study. BMC Health Services Research, 18(1), 634. https://doi.org/10.1186/s12913-018-3365-6

Lorenc, T., Petticrew, M., Welch, V., \& Tugwell, P. (2013). What types of interventions generate inequalities? Evidence from systematic reviews. Journal of Epidemiology and Community Health, 67(2), 190-193. https://doi.org/10.1136/jech-2012-201257

Martínez-Salgado, C. (2012). El muestreo en investigación cualitativa: principios básicos y algunas controversias. Ciência \& Saúde Coletiva, 17(3), 613-619. https://doi.org/bgqn MacMillan, H. L., Jamieson, E., Nadine-Wathen, C., Boyle, M. H., Walsh, C. A., Omura, J., Walker, J. M., \& Lodenquai, G. (2007). Development of a policy-relevant child maltreatment research strategy. The Milbank Quarterly, 85(2), 337-374. https://doi.org/b29jbx 
Ministerio de Salud. (2017). Plan Nacional de Salud Mental 2017-2025. Ministerio de Salud de Chile.

Ministerio de Salud. (2018). Programa nacional de salud integral de adolescentes y jóvenes: nivel especializado de atención abierta y cerrada 2018. Ministerio de Salud de Chile.

Minoletti, A., Rojas., G., \& Horvitz-Lennon, M. (2012). Salud mental en atención primaria en Chile: aprendizajes para Latinoamérica. Cadernos Saúde Coletiva, 20(4), 440-447. https://doi.org/10.1590/s1414-462X2012000400006

Nelson, C. A., Zeanah, C. H., \& Fox, N. A. (2019). How early experience shapes human development: The case of psychosocial deprivation. Neural Plasticity, 2019, 1-12. https://doi.org/10.1155/2019/1676285

Olabarría, B., \& Mansilla, F. (2007). Ante el burnout: cuidados a los equipos de salud mental. Revista de Psicopatología y Psicología Clínica, 12(1), 1-14. https://doi.org/f6vh

Petrowski, N., Cappa, C., \& Gross, P. (2017). Estimating the number of children in formal alternative care: Challenges and results. Child Abuse \& Neglect, 70, 388-309.

https://doi.org/10.1016/j.chiabu.2016.11.026

Rozanski, C. (2003). Abuso sexual infantil ¿Denunciar o silenciar? Ediciones B.

Sánchez-Reyes, J. E., Cantor-Jiménez, J., Castro-Sardi, X., \& Bolaños, Y. (2019). Concepciones de niño y modos de relación de cuidadores y profesionales con niños institucionalizados, Colombia. Revista Latinoamericana de Ciencias Sociales, Niñez y Juventud, ${ }_{17}(2), 1-24$. https://doi.org/10.11600/1692715x.17209

Santana, A., \& Farkas, Ch. (2007). estrategias de autocuidado en equipos profesionales que trabajan en maltrato infantil. Psykhe, 16(1), 77-89. https://doi.org/b2tpsj

Santos, D. B. C., Vázquez-Ramos, V., Oliveira, C. da C. C., \& López-Arellano, O. (2019). Accesibilidad en salud: revisión sobre niños y niñas con discapacidad en BrasilPerú-Colombia. Revista Latinoamericana de Ciencias Sociales, Niñez y Juventud, $17(2)$, 1-20. https://doi.org/10.11600/1692715x.17206

Scharager, J., \& Molina, M. L. (2007). El trabajo de los psicólogos en los centros de atención primaria del sistema público de salud en Chile. Revista Panamericana de Salud Pública, 22, 149-59. https://doi.org/10.1590/s1020-49892007000800001

Sempik, J., Ward, H., \& Darker, I. (2008). Emotional and behavioral difficulties of children and young people at entry into care. Clinical Child Psychology and Psychiatry, 13(2), 221-233. https://doi.org/10.1177/1359104507088344

Soneira, A. J. (2006). La teoría fundamentada en los datos (Grounded Theory) de Glaser y Strauss. Gedisa. 
Ståhlberg, O., Anckarsäter, H., \& Nilsson, T. (2010). Mental health problems in youths committed to juvenile institutions: Prevalences and treatment needs. European Child \& Adolescent Psychiatry, 19, 893-903. https://doi.org/10.1007/s00787-010-0137-1

Strauss, A., \& Corbin, J. (2002). Bases de la investigación cualitativa: técnicas y procedimientos para desarrollar la teoría fundamentada. Editorial Universidad de Antioquia.

Vicente, B., Saldivia, S., \& Kohn, R. (2012). Epidemiology of mental disorders, use of service, and treatment gap in Chile. International Journal of Mental Health, 41(1), 7-20. https://doi.org/10.2753/imhoo20-7411410101

Vilà, M., Cruzate, C., Pernasb, F., Creixell, J., González, M. P., \& Davins, J. (2015). Burnout y trabajo en equipo en los profesionales de Atención Primaria. Atención Primaria, 47(1), 25-31. https://doi.org/10.1016/j.aprim.2014.01.008

Zachik, A. A., Naylor, M. W., \& Klaehn, R. L. (2010). Child and adolescent psychiatry leadership in public mental health, child welfare, and developmental disabilities agencies. Child and Adolescent Psychiatric Clinics, 19(1), 47-61. https://doi.org/c5cm7q 